\title{
EXTENSIONS OF CUTTING PROBLEMS: SETUPS*
}

\author{
Sebastian Henn and Gerhard Wäscher**
}

Received May 3, 2013 / Accepted July 19, 2013

\begin{abstract}
Even though the body of literature in the area of cutting and packing is growing rapidly, research seems to focus on standard problems in the first place, while practical aspects are less frequently dealt with. This is particularly true for setup processes which arise in industrial cutting processes whenever a new cutting pattern is started (i.e. a pattern is different from its predecessor) and the cutting equipment has to be prepared in order to meet the technological requirements of the new pattern. Setups involve the consumption of resources and the loss of production time capacity. Therefore, consequences of this kind must explicitly be taken into account for the planning and control of industrial cutting processes. This results in extensions to traditional models which will be reviewed here. We show how setups can be represented in such models, and we report on the algorithms which have been suggested for the determination of solutions of the respective models. We discuss the value of these approaches and finally point out potential directions of future research.
\end{abstract}

Keywords: cutting, problem extensions, setups, exact algorithms, heuristic algorithms, multi-objective optimization.

\section{INTRODUCTION}

The body of literature on cutting and packing $(\mathrm{C} \& \mathrm{P})$ problems is still rapidly growing. In order to provide an instrument for the systematic organisation and categorisation of existing and new literature in the field, Wäscher et al. (2007) introduced a typology of C\&P problems in which the criteria for the definition of homogeneous problem categories are related to "pure" problem types, i.e. problem types of which a solution comprises information on the set of cutting/packing patterns, the number of times they have to be applied, and the corresponding objective function value, only.

Cutting and packing problems from practice, on the other hand, are often tied to other problems and cannot be separated from them without ignoring important mutual interdependencies. Realistic solution approaches to such integrated problems, therefore, do not only have to provide

*Invited paper

**Corresponding author

Otto-von-Guericke University Magdeburg, Faculty of Economics and Management - Management Science - Postbox 4120, 39016 Magdeburg, Germany. E-mail: gerhard.waescher@ovgu.de 
cutting patterns, but also have to deal with additional problem-relevant aspects such as processing sequences (as in the pattern sequencing problem; cf. Foerster \& Wäscher, 1998; Yanasse, 1997; Yuen, 1995; Yuen \& Richardson, 1995), due dates (cf. Helmberg, 1995; Li, 1996; Reinertsen \& Vossen, 2010; Rodriguez \& Vecchietti, 2013), or lot-sizes ( $c f$. Nonås \& Thorstenson, 2000). In this paper, we address the issue of setups which arise in industrial cutting processes whenever a new cutting pattern is started (i.e. a pattern is different from its predecessor) and the cutting equipment has to be prepared in order to meet the technological requirements of the new pattern. Setups of this kind involve the loss of production time capacity and the consumption of resources which can be avoided or, at least, reduced by taking into account theses consequences when decisions are made on the set of cutting patterns to be applied.

Setups represent an important issue of real-world (extended) cutting problems but have only been considered occasionally in the literature related to C\&P problems. We, therefore, would like to give an overview of the state-of-the-art in the field, but also to emphasize theoretical deficiencies and future research opportunities. We restrain our analysis to papers which are publicly available and have been published in English in international journals, edited volumes, and conference proceedings between by the end of 2012 .

The remaining part of this paper is organized as follows: In Section 2 we will be dealing with the fundamentals of (extended) C\&P problems. The general structure of C\&P problems will be introduced and, in order to motivate the topic of this paper, we will describe situations in which cutting problems should be considered in combination with setups. Most of the papers dealing with setups can be seen as an extension of the so-called Single Stock Size Cutting Stock Problem (SSSCSP), as it has been defined in the typology of Wäscher et al. (2007). The problem is of the input minimization type. It will be formulated in Section 3, and it will be shown how it can be modeled and solved. A straightforward approach for the integration of setups into the SSSCSP would be to transform this model into a total cost minimization model which comprises the cost of the input and the cost of setups. How this can be achieved, is demonstrated in Section 4. In this section, we also review the literature which concerns corresponding solution algorithms. In practice, however, often the values of the cost coefficients which are necessary to formulate such a total cost minimization model are not known (Diegel et al., 1996b, p. 636; Vasko et al., 2000, p. 9). In that case one has to switch to auxiliary models, which - instead of referring to the total cost - evaluate the quality of solutions by means of two "cost drivers", namely input quantity or amount of trim-loss on one hand, and number of setups on the other. The methods presented in Sections 5 and 6 can be characterized as lexicographic approaches. Section 5 deals with the minimization of input or trim loss subject to a given number of setups, Section 6 with the minimization of the number of setups subject to a given amount of input or trim-loss. A more general approach, multi-objective optimization, is considered in Section 7. In particular, we describe methods, which aim at the provision of a single (compromise) solution, and methods which try to give a more detailed view of the trade-off between the cost drivers. Section 8 discusses the adequacy of the previously presented approaches for the solution of cutting problems with setups from industrial practice. Finally, in Section 9, the paper concludes with an outlook on research opportunities, i.e. research areas which have not or only rudimentarily been dealt with so far. 


\section{FUNDAMENTALS}

\subsection{Cutting and Packing Problems - Definition and Typology}

The general structure of cutting and packing problems can be summarised as follows (in the following, in particular $c f$. Wäscher et al., 2007):

Given are two sets of elements, namely

- a set of large objects (supply) and

- a set of small items (demand),

which are defined exhaustively in one, two, or three (or an even larger number) of geometric dimensions. Select some or all small items, group them into one or more subsets and assign each of the resulting subsets to one of the large objects such that the geometric condition holds, i.e. the small items of each subset have to be laid out on the corresponding large object such that

- all small items of the subset lie entirely within the large object and

- the small items do not overlap,

and a given objective function is optimised.

With respect to standard problems, basically two types of assignment can be distinguished. In the case of input (value) minimization, the set of large objects is sufficient to accommodate all small items. All small items are to be assigned to a selection (a subset) of the large object(s) of minimal value. There is no selection problem regarding the small items.

In the case of output (value) maximisation, the set of large objects is not sufficient to accommodate all the small items. All large objects are to be used, to which a selection (a subset) of the small items of maximal value has to be assigned. There is no selection problem regarding the large objects.

Depending on the specific problem environment, the "value" of objects/items has to be defined more precisely and may be represented by costs, revenues, or material quantities. Often, the value of the objects/items can be assumed to be directly proportional to their size such that the objective function considers length (one-dimensional problems), area (two-dimensional problems), or volume (three-dimensional problems) maximization (output) or minimization (input). In such cases, both "output (value) maximization" and "input (value) minimization" may be replaced by "waste minimization", i.e. the minimization of the total size of unused parts of the (selected) large objects. In the environment of cutting problems often the term "trim-loss minimization" is used.

The C\&P problems dealt with in this paper are exclusively related to the planning and control of industrial cutting processes (cutting problems). Therefore, any unique algebraic or graphical representation of an assignment of small items to a large object will be referred to as a cutting pattern. Each solution to a cutting problem consists (at least) of a cutting plan, i.e. a set of cutting patterns and the corresponding application frequencies. 
The determination of cutting patterns and the corresponding application frequencies represent the core elements in solving cutting problems. Despite of having the above-described problem structure in common, actual cutting problems may be quite different in detail and do not allow for being treated by the same or even similar solution approaches. In order to define more homogeneous problem types, Wäscher et al. (2007) - apart from "kind of assignment" use two additional criteria, namely", "assortment of large objects" and "assortment of small items", in order to identify 14 (intermediate) problem types. By means of two additional criteria, "dimensionality" and "shape of small items" further refined problem types can be obtained.

\subsection{Setups}

Realistic solution approaches to cutting problems in practice do not only have to provide cutting plans, but also have to deal with additional aspects and have to answer additional questions. One such aspect concerns the setups which may arise in industrial cutting processes whenever a new cutting pattern different from its predecessor is started and the cutting equipment has to be prepared in order to meet the technological requirements of the new pattern. Setups of this kind involve the loss of production time capacity and the consumption of resources.

- In the paper industry, reels in sizes demanded by customers are to be cut from large paper rolls (jumbos). This is carried out on a so-called winder which unwinds a jumbo and rewinds it on cores while the paper is being slit. The slitting itself is done by means of rotating knifes or laser beams which have to be repositioned whenever a new cutting pattern is started. In order to do so, the winder has to be stopped and the paper has to be fed in again. After the winder has been restarted the paper may take some time to settle in, during which defects can occur (cf. Diegel et al., 2006, p. 708f.).

- Kolen \& Spieksma (2000) describe a similar setting related to the production of abrasives. Abrasives are also manufactured in rolls (raws) which have to be cut down into rolls of smaller widths ordered by external customers. Apart from minimizing the trim loss, the company also seeks to minimize the number of different cutting patterns since the cutting machine undergoes a setup every time two consecutive raws have to be cut according to different patterns. Setting up the machine is a manual, time-consuming operation. The authors mention that a setup might require up to three quarters of an hour.

- In the corrugated cardboard industry, a similar technology (rotating knifes) is used for slitting the final product into smaller widths (demanded by external customers or required by subsequent internal production stages). The slitting of the cardboard follows immediately after the actual production process. The production process itself is a continuous one and cannot be interrupted, i.e. while the knifes are being repositioned for a new cutting pattern, cardboard keeps shooting out of the production facility without being cut. It can only be treated as waste and has to be disposed at additional costs ( $c f$. Pegels, 1967; Haessler \& Talbot, 1983).

- In the steel industry, a cutting machine cuts steel strips or plates required by customers from larger slabs, coils, bars ( $c f$. Vasko et al., 1999), or from an endless steel tube ( $c f$. 
Hajizadeh \& Lee, 2007). The machine operates a number of knives in parallel or successively after one another. Typically, the entire cutting process needs to be stopped in order to change the complete set or a subset of the used knives, or some knife positions.

Aiming entirely at a minimization of input, it cannot be expected that traditional approaches to cutting processes will always give satisfactory results under these conditions since input minimization on one hand and setup minimization on the other are conflicting goals ( $c f$., among others, Farley \& Richardson, 1984, p. 246; Vanderbeck, 2000, p. 916; Umetani et al., 2003a, p. 1094; Umetani et al., 2006, p. 45; Cui et al., 2008, p. 677; Moretti \& de Salles Neto, 2008, p. 63). Instead, it would be useful to reduce the number of cutting patterns (and, by doing so, the number of setups) in a solution at the expense of additional input or trim loss if that would result in a reduction of the respective total costs. Consequently, the inclusion of setups represents a straightforward extension to the traditional input minimization models.

\section{THE SINGLE STOCK SIZE CUTTING STOCK PROBLEM (SSSCSP)}

Of all 14 intermediate problem types identified by Wäscher et al. (2007), the Single Stock Size Cutting Stock Problem (SSSCSP) is of particular interest, because the literature to be reviewed here is related to problems of this kind in the first place. In this section, the SSSCSP will be defined and it will be shown how it can be modeled. The models which will be presented are general in the sense that they represent one-dimensional, two-dimensional and three-dimensional cutting problems with small items of any shape.

\subsection{Problem Formulation}

The SSSCSP can be stated as follows: A weakly heterogeneous assortment of small items has to be cut from a stock of large objects, which are available in a sufficiently large number of identical pieces of a single given (standard) size. The number of the large objects needed to provide all small items has to be minimized.

\subsection{Two Models for the SSSCSP}

In the following, we introduce the notation for a formal representation (model) of the SSSCSP.

$I: \quad$ set of all small item types which have to be cut, $I=\{1,2, \ldots, m\}$;

$J: \quad$ set of all (relevant) cutting patterns which can be applied to the large object type in order to cut it down into small items;

$a_{i j}$ : number of times small item type $i(i \in I)$ appears in cutting pattern $j$ $(j \in J)$;

$d_{i}$ : demand for small item type $i(i \in I)$; number of times small item type $i(i \in I)$ has to be provided;

$t_{j}: \quad$ trim loss per large object involved with cutting pattern $j(j \in J)$;

$x_{j}$ : number of large objects which have to be cut according to cutting pattern $j(j \in J)$; number of times cutting pattern $j(j \in J)$ is applied (application frequency of cutting pattern $j(j \in J))$. 
By means of these symbols, the SSSCSP can then be represented by model M 1.1 of Table 1, which we call the Input Minimization Model of the SSSCSP (IMM) here. The objective function (3.1) postulates a minimization of the total number of large (stock) objects (input minimization) which is necessary to satisfy all demands. Demand constraints (3.2) guarantee that (at least) the demanded number of items of each small item type is provided. Integer constraints (3.3) make sure that large objects are always cut down completely.

Waste may occur as trim loss contained in the selected cutting patterns (i.e. parts of a large object which are not covered by small items), but also as excess production (surplus) which is provided beyond the actual demand of the small item types. Since all demands are fixed and have to be satisfied exactly, input minimization and waste minimization are equivalent goals.

All large objects are identical, not only with respect to their size but also with respect to their material cost. Therefore, minimization of the material (input) quantity (i.e. minimization of the number of large objects) will also result in a minimization of the total material (input) cost.

Alternatively, instead of input minimization, one may also chose trim loss minimization as a goal. The corresponding model is given by M 1.2 (TMM: Trim Loss Minimization Model of the SSSCSP) of Table 1. The objective function (3.4) calls for a minimization of the total trim loss. Since, according to constraints (3.5), the small item types have to be provided exactly in the demanded quantities, no excess production can occur. In other words, a trim loss minimal solution of (3.5) and (3.6) is also a waste minimal solution. Consequently, M 1.1 and M 1.2 are equivalent models. Optimal solutions of M 1.1 on one hand and of M 1.2 on the other will possess the same amount of input and the same amount of waste, even though not necessarily the same amount of trim loss.

Table 1 - Basic Optimization Models for the SSSCSP.

\begin{tabular}{|c|c|c|}
\hline \multicolumn{3}{|c|}{ Basic Optimization Models } \\
\hline \multicolumn{3}{|c|}{ Input Minimization } \\
\hline (M 1.1) IMM & & \\
\hline$\sum_{j \in J} x_{j} \rightarrow \operatorname{Min} !$ & & $(3.1)$ \\
\hline$\sum_{j \in J} a_{i j} \cdot x_{j} \geq d_{i}$ & $i \in I$ & $(3.2)$ \\
\hline$x_{j} \geq 0$, integer, & $j \in J$ & $(3.3)$ \\
\hline \multicolumn{3}{|c|}{ Trim Loss Minimization } \\
\hline (M 1.2) TMM & & \\
\hline$\sum_{j \in J} t_{j} \cdot x_{j} \rightarrow \operatorname{Min} !$ & & $(3.4)$ \\
\hline$\sum_{j \in J} a_{i j} \cdot x_{j}=d_{i}$ & $i \in I$ & $(3.5)$ \\
\hline$x_{j} \geq 0$, integer, & $j \in J$. & $(3.6)$ \\
\hline
\end{tabular}


We note that, due to the fact that excess production is permitted, with respect to model M 1.1, it is sufficient to consider complete patterns (i.e. patterns to which no further small item can be added) only in the set $J$ of cutting patterns, while in M 1.2 - due to the equations in (3.5) $-J$ must also include all incomplete patterns. The same applies to model M 1.1 if the demand constraints (3.2) are formulated as equations (as, e.g. in Vanderbeck, 2000, p. 915).

Goulimis (1990, p. 203) argues that equality-constrained optimization models are more difficult to solve and tend to give patterns with trim loss unacceptable in practice. Therefore, he and several other authors (e.g. Haessler, 1975, 1988; Vasko et al., 2000) allow for demand tolerances, i.e. demands which only have to be satisfied within certain pre-specified limits, instead of demands which have to be satisfied exactly. Constraints (3.5) are replaced by constraints of the type

$$
\min \left(d_{i}\right) \leq \sum_{j \in J} a_{i j} \cdot x_{j} \leq \max \left(d_{i}\right), \quad j \in J
$$

From an economist's point of view, this is not totally convincing. Since both input and output of the cutting process are now variable, it would formally be necessary to introduce profit maximization as a goal in the corresponding objective function. Such modifications are not common in the literature, though. Among the few exceptions is a paper of Schilling \& Georgiadis (2002), who develop an even more general model which does not only comprise the revenues from the provided small items (final products), the cost of the large objects (material cost) and the cost of setups, but also explicitly considers the cost of disposing the trim loss.

We would also like to point out that further modeling approaches to the SSSCSP exist, namely the one-cut model of Rao (1976) and Dyckhoff (1981) and the network flow model of Valério de Carvalho (1998). We do not present any further details of these models here, since they are less suitable for being extended for the inclusion of setup aspects.

\subsection{Solution Approaches}

When given a particular problem instance of the SSSCSP, one may immediately think of generating the respective model M 1.1 or M 1.2 and have it solved by a commercial LP solver. This is, in fact, a solution approach which is occasionally taken in practice when the number of item types is small and/or when special constraints of the cutting technology limit the number of feasible cutting patterns to a small number ( $c f$. Koch et al., 2009). In general, this approach will not be feasible, though, since the number of cutting patterns and, likewise, the number of variables in the corresponding model, grows exponentially with the number of different item types and very easily reaches prohibitive dimensions.

The described limitations of explicit model formulation may be overcome by column generation techniques which have already been developed by Gilmore \& Gomory $(1961,1963,1965)$ in the early 1960s. By means of these techniques, at first a solution of the continuously relaxed model M 1.1 or M 1.2 is computed, which is usually not all-integer and, thus, must then be converted into an integer solution in a second step. This step is a problematic one with respect to the issue which is highlighted in this paper, namely the inclusion of setup considerations. A 
straightforward approach for the generation of an integer solution consists of rounding up the non-integer application frequencies of the cutting patterns to the next integer. This immediately gives a feasible solution to the original problem (as stated in Section 3.1) while rounding down does not, since demand constraints will be violated. The drawback of this simple procedure lies in the fact that the true input-minimal or trim loss-minimal solution will usually be missed, probably by far. On the other hand, by means of Branch-and-Bound or sophisticated rounding techniques (Wäscher \& Gau, 1996) applied to the non-integer solution, an input-minimal or trim loss-minimal solution can be identified, but usually at the expense of additional cutting patterns/setups in the solution.

Thus it is not surprising that when one has to deal with setups, certain heuristic approaches are often used which have been popular in the early days of applying operations research methods to cutting problems and which have been proven flexible enough to incorporate additional practical requirements. Methods of this type are usually based on the Repeated Pattern Exhaustion Technique (RPE Technique; Dyckhoff, 1988) for the (heuristic) solution of cutting problems. This is a sequential solution approach (usually described for the one-dimensional SSSCSP) in which cutting patterns and the frequencies according to which they are admitted into the cutting plan are determined successively. It consists of three phases: (1) generation of an "acceptable" cutting pattern $j,(2)$ determination of the maximal frequency $x_{j}$ according to which the pattern can be applied (limited by the remaining demands), and (3) update of the demands. These phases are repeated until all demands are satisfied ( $c f$. Pierce, 1964, p. 28ff.). Integrality of the generated solution can be guaranteed easily in step 3 .

\section{TOTAL COST MINIMIZATION FOR THE SSSCSP WITH SETUPS}

\subsection{Total Cost Models}

The IMM can be extended in a straightforward way in order to take into account for setups by switching to an objective function which aims at minimizing the total decision-relevant cost. We introduce the following additional symbols:

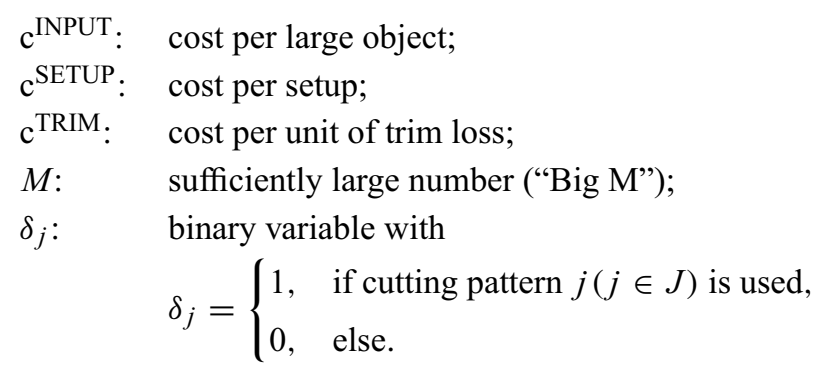

Model M 2.1 of Table 2 represents the Input-Based Total Cost Minimization Model of the SSSCSP with Setups (IB_TCMM_SU; $c f$. Farley \& Richardson, 1984, p. 246; Jardim Campos \& Maculan, 1995; Diegel et al., 1996b; Foerster, 1998, p. 75; Moretti \& de Salles Neto, 2008, p. 62f.; Golfeto et al., 2009a, p. 367). 
Table 2 - Total Cost Minimization Models of the SSSCSP with Setups.

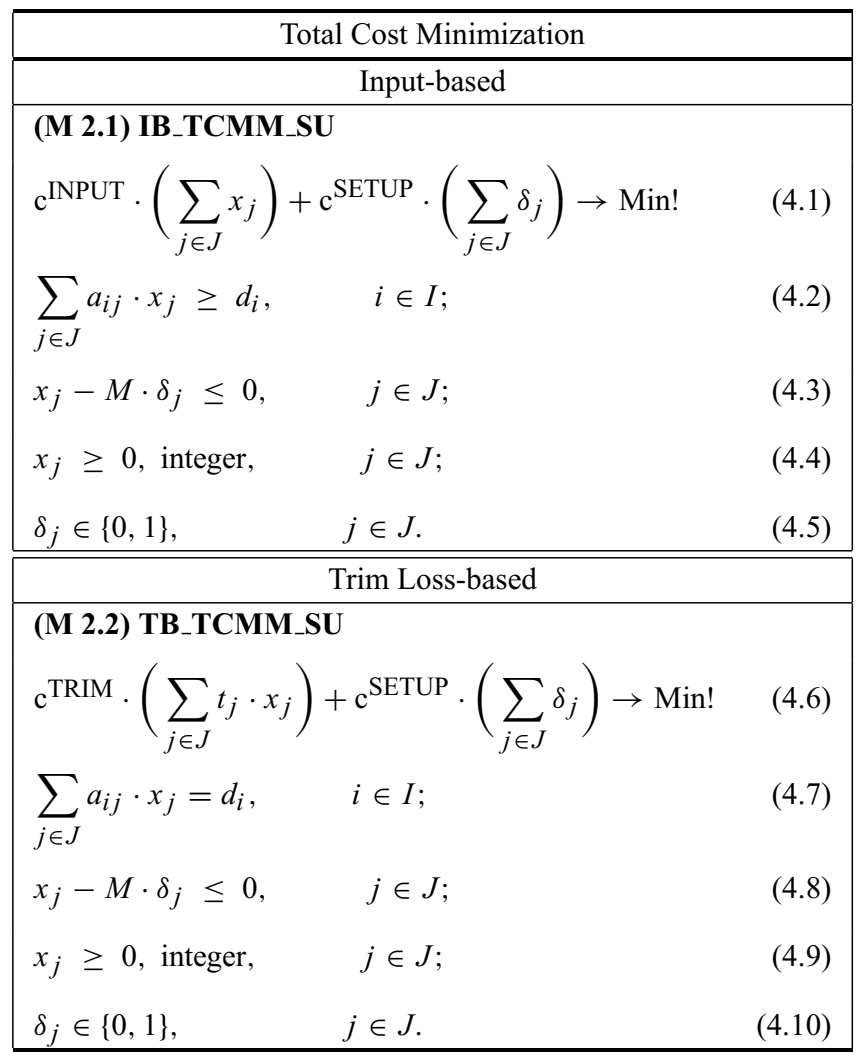

For any solution of the optimization model M 2.1, $\sum_{j \in J} \delta_{j}$ will give the number of cutting patterns and, equivalently, the number of setups. Consequently, the objective function (4.1) now includes the total cost of the utilized large objects (input cost; basically consisting of material cost) and the total cost of setups. Obviously, an implicit assumption of this model is that the cost for setting up the cutting equipment is proportional to the number of setups, but independent from the respective pattern and also from the preceding and the succeeding pattern. (4.8) are auxiliary constraints in which $\mathrm{M}$ is a sufficiently large number. Each of these constraints - in combination with the minimization instruction for the objective function value - guarantees that the binary variable $\delta_{j}$ corresponding to cutting pattern $j(j \in J)$ is set to one and the setup costs are included in the calculation of the objective function value whenever cutting pattern $j$ is actually used.

Likewise, one may also take the TMM as the basic model and extend it with respect to the number of cutting patterns. This gives rise to the Trim Loss-Based Total Cost Minimization Model of the SSSCSP with Setups (TB_TCMM_SU; cf. Haessler, 1975, p. 486; Haessler, 1988, p. 1461; Vasko et al., 2000, p. 9; Golfeto et al., 2009b). The objective function includes the total cost of trim loss (material cost, cost of disposal) and the total cost of setups. We remark that M 2.2 can also be taken as a modeling concept when costs are to be considered which are dependent on 
the cutting pattern applied to a large object. In that case, in the objective function the constant $\mathrm{c}^{\text {INPUT }}$ has to be omitted and the $t_{j}$-coefficients have to be replaced by the corresponding costs $c_{j}$ related to cutting one large object according to pattern $j$.

\subsection{Solution Approaches}

The solution approaches to be presented here are all of the heuristic type. Unlike the methods to be discussed in the subsequent sections, they all assume that the relevant cost information is available and make explicitly use of this information for the generation of solutions.

Farley \& Richardson (1984) present a heuristic for the two-dimensional TB_TCMM_SU which is based on the column-generation approach by Gilmore \& Gomory (1965). It starts from an optimal solution of the continuous relaxation of the SSSCSP and, in a series of iterations, tries to reduce the number of structural variables (i.e. the number of different patterns) in the solution. Unlike in the Gilmore-Gomory approach, variables corresponding to cutting patterns are not discarded as they are leaving the basis. The authors give (heuristic) rules according to which basic structural variables and non-basic slack variables should be swapped. The method generates a sequence of feasible, not necessarily integer basic solutions and terminates when no improved or feasible solution can be found.

The authors have evaluated the proposed method on fifty two-dimensional problem instances with twenty small item types each. For (relative) values of the pattern cost (cost of trim loss per pattern) over the setup cost ranging from 1:0 up to 1:20 they demonstrated how the proportion between waste and number of patterns changed in the respective best solution. The question of generating integer solutions was not specifically addressed. In principle, the proposed method can also be applied to the one- and three-dimensional SSSCSP with identical setups.

Jardim Campos \& Maculan (1995) consider the one-dimensional IB_TCMM_SU. They drop the integer constraints (4.4) for the $x_{j}$ variables and derive the Langrangian dual of the remaining optimization problem. This problem may be solved by subgradient methods into which the column-generation technique of Gilmore \& Gomory $(1961,1963)$ has been integrated. No numeric example is given and no computational experience is reported. The question of how integer solutions can be generated is not addressed.

Diegel et al. (1996b) discuss an extensive numerical example for the one-dimensional IB_TCMM_SU. On the basis of an explicit model formulation they exemplify the necessity to consider setups in the planning process and discuss how appropriate (relative) cost factors (pattern costs, setup costs) can be chosen when the exact ones are not known. They do not address any computational aspects related to problem instances which result in models too large for being solved by commercial optimizers, or for which the explicit models cannot even be generated (because they contain too many columns).

Moretti \& Salles Neto (2008) adopt the classic column generation approach of Gilmore \& Gomory (1961) for the (continuously relaxed) one-dimensional IB_TCMM_SU. The master problem consists of an objective function, which is a linearization of (4.1), and a set of cutting patterns, 
which establishes a (column) basis of the coefficient matrix of the constraint system (3.2). It is solved by means of an augmented Lagrangian method. The pricing problem consists of a bounded knapsack problem; the respective simplex multipliers are determined by solving another linear optimization problem of the type (3.4)-(3.6), where the coefficients $t_{j}$ are computed from the values of $\mathrm{c}^{\text {INPUT }}$ and $\mathrm{c}^{\text {SETUP }}$, and the application frequencies $x_{j}$ of the current solution of the master problem. It is solved in order to determine a new column (cutting pattern) which would improve the current objective function value of the master problem. If such a column is identified, it enters the basis and the master problem is solved again. The solution of the master problem is recorded if it is better - with respect to (4.1) - than the solutions generated from the master problem in previous iterations. These steps are repeated until the pricing problem provides no further column that would improve the current solution of the master problem. The initial set of cutting patterns is determined by the Sequential Heuristic Procedure of Haessler (1975). Integer solutions are obtained from the best recorded solution by application of the BRURED rounding procedure (Neumann \& Morlock, 1993, p. 431f.; Wäscher \& Gau, 1996, p. 134), which may result in excess production but does not increase the number of cutting patterns in the solution.

The authors have tested their approach (NANLCP) on a set of randomly generated problem instances from Foerster \& Wäscher (2000), which includes 18 problem classes with 100 instances each. For $\mathrm{c}^{\text {INPUT }}=1$ and $\mathrm{c}^{\text {SETUP }}=5$ they found that NANLCP obtained solutions with lower average total cost than KOMBI234 (i.e. the method proposed by Foerster \& Wäscher, 2000) does for 15 problem classes. For $\mathrm{c}^{\text {INPUT }}=1$ and $\mathrm{c}^{\text {SETUP }}=10$ NANLCP outperformed KOMBI234 for all 18 problem classes. On the other hand, computing times for NANLCP were significantly larger than for KOMBI234 and seem to represent a major drawback of the proposed method (cf. Moretti \& Salles Neto, 2008, p. 76f.) We further note, that KOMBI234 is a method based on the (implicit) assumption that $\mathrm{c}^{\text {INPUT }} \gg \mathrm{c}^{\text {SETUP }}$ (see below). Therefore, we conclude that the results from the numerical experiments with NANLCP on one hand, and with KOMBI234 on the other cannot really be compared to each other.

Golfeto et al. (2009a) introduce a symbiotic genetic algorithm (SGA) for the one-dimensional IB_TCMM_SU which works with two populations, a population of solutions (cutting plans) and a population of cutting patterns. The chromosome of each individual from the population of solutions consists of pairs of which one entry corresponds to a pattern from the population of cutting patterns, while the other represents the respective frequency of application. Mutation and uniform crossover operators are used to generate new solutions. An elitist strategy is applied for the selection of individuals for the next generation. The fitness function consists of the objective function (4.1) and two additional terms. The first one is a measure of the relative trim loss, and the second one a penalty if the solution is not feasible. The chromosome of an individual of the cutting pattern population consists of a list, in which each entry represents an item type included in the pattern. Mutation and two-point crossover operators are applied in order to produce new patterns. The calculation of the fitness of a pattern is based on whether it is included in an elite list of the solutions. If this is the case, the fitness of the pattern is increased according to the position of the solution in the elite list. By doing so, priority is given to patterns which appear in 
the best solutions. In the evolutionary process these patterns are meant to generate other patterns which will provide even better solutions.

The authors have run numerical experiments similar to the ones of Moretti and Salles Neto (2008). In particular, the 18 classes of randomly generated problem instances from Foerster \& Wäscher (2000) were considered again. For $\mathrm{c}^{\text {INPUT }}=1$ and $\mathrm{c}^{\text {SETUP }}=1$ they found that the SGA outperformed KOMBI234 on one and NANLCP on seven problem classes. For $\mathrm{c}^{\mathrm{INPUT}}=1$ and $\mathrm{c}^{\mathrm{SETUP}}=5$, in comparison to KOMBI234, the SGA provided smaller average costs per problem instance for ten problem classes, and in comparison to NANLCP for four classes. For $\mathrm{c}^{\text {INPUT }}=1$ and $\mathrm{c}^{\text {SETUP }}=10$ better solutions (on average) were found for 14 problem classes (KOMBI234) and six classes (NANLCP), respectively. The computing times were significantly larger than those for KOMBI234 (with the exception of one problem class) and NANLCP, and ranged from 17.66 to 426.08 seconds per instance (for $\mathrm{c}^{\mathrm{INPUT}}=1, \mathrm{c}^{\mathrm{SETUP}}=5$ ) on a AMD SEMPRON 2300+ PC (1.5 MHz, $640 \mathrm{MB}$ RAM). We remark that the numerical experiments also included a comparison of the SGA to the ILS approach of Umetani et al. (2006) and to the heuristic of Yanasse \& Limeira (2006). We do not report the results here since we believe that, again, these methods belong to different classes of methods (see below) and, thus, the results are not really comparable.

Atkin \& Özdemir (2009) refer to a one-dimensional cutting problem in coronary stent manufacturing. The proposed solution approach consists of two phases. In the first phase, a heuristic is applied in order to generate a set of trim-loss minimal cutting patterns. In the second phase, these patterns are used for setting up a model of the IB_TCMM_SU type from which the respective frequencies of application are determined. The model is more complex than the above-presented one. Apart from input (material) and setup costs, it also incorporates labor cost for regular working hours and overtime, and costs of tardiness. By means of numerical experiments the authors demonstrated that - under conditions encountered in practice - the heuristic from the first phase already gives satisfactory results when used as a stand-alone procedure and, thus, may not necessarily be accompanied by the succeeding linear programming approach form the second phase.

\section{INPUT OR TRIM LOSS MINIMIZATION SUBJECT TO SETUP LIMITATIONS}

Given that the cost coefficients of the total cost models M 2.1 and M 2.2, namely the cost per large object $\mathrm{c}^{\text {INPUT }}$, the cost per unit of trim-loss $\mathrm{c}^{\text {TRIM }}$ and the cost per setup $\mathrm{c}^{\text {SETUP }}$, are not always known in practice, instead of minimizing the total cost as defined in (4.1) and (4.6) one may turn to limiting one of the underlying cost drivers to a specific maximal amount while the amount of other one is tried to be kept as small as possible. In the following, we will first deal with minimizing the input or the trim loss subject to a given number of setups.

\subsection{Setup-Constrained Input or Trim Loss Minimization Models}

Let the following additional notation be introduced:

$\max (s)$ : maximal number of setups/cutting patterns permitted in a solution. 
Then, on the basis of the IB_TCMM_SU, a Setup-Constrained Input Minimization Model of the SSSCSP with Setups (SC_IMM_SU) can be obtained if we replace objective function (4.1) in Model M 2.1 by

$$
\sum_{j \in J} x_{j} \rightarrow \operatorname{Min} !
$$

and add the following constraint

$$
\sum_{j \in J} \delta_{j} \leq \max (s)
$$

which limits the number of cutting patterns in the solution to an acceptable number $\max (s)$. Umetani et al. (2003a, p. 1094) also call this problem the Pattern-Restricted Version of the Cutting Stock Problem.

Likewise, a Setup-Constrained Trim Loss Minimization Model of the SSSCSP with Setups (SC_TMM_SU) can be formulated if - in Model M 2.2 - the objective function (4.6) is replaced by

$$
\sum_{j \in J} t_{j} \cdot x_{j} \rightarrow \operatorname{Min} !
$$

and constraint (5.2) is added.

\subsection{Solution Approaches}

Umetani et al. (2003a) introduce an Iterated Local Search (ILS) Algorithm for the one-dimensional SC_IMM_ SU. It starts from an initial solution with $\max (s)$ cutting patterns which is generated by means of a modified First-Fit-Decreasing (FFD) Method for the Bin-Packing Problem. Then a simple local search is applied in which the algorithm continues to replace the current set of patterns with an improved one (consisting of the same number of patterns) from the respective neighborhood until no better set is found. The neighborhood (called 1-add neighborhood in a subsequent paper; $c f$. Umetani et al., 2006) of a solution is defined by the set of patterns which can be obtained by increasing the number of one piece type in a cutting pattern while reducing the number(s) of one or several other piece types in the same pattern such that the feasibility of the pattern is maintained. In order to direct the search for an improved set of solutions, at each iteration the dual of the LP relaxation of (3.1)-(3.3) (consisting of the current set of patterns only) is solved because the optimal values of the dual variables can be seen as an indication of how effective it will be to increase the number of times item type i appears in pattern $j$. The frequencies of the patterns in the new set are determined by solving the corresponding continuous relaxation of the integer programming problem (3.1)-(3.3) and applying a simple rounding technique to obtain integrality. Since it is necessary to solve (the LP relaxation of) this problem for many neighbors of each solution, the authors apply a variant of the Simplex Method at this stage which incorporates the Criss-Cross-Method (cf. Terlaky, 1985). Pivoting operations can be carried out from the optimal tableau of the previous iteration, reducing the number of required pivoting operations significantly. After having reached a local optimum, the simple local search is repeated by applying it to new initial solutions. These new initial solutions are obtained by swapping one item from a pattern in the currently best solution with one or several items from 
another pattern. The ILSA terminates if no improvement has been obtained for a pre-specified number of iterations.

The authors have performed numerical experiments in which the ILSA was evaluated in comparison to Haessler's RPE Technique (SHP) and KOMBI234 on the 18 classes of randomly generated problem instances of Foerster \& Wäscher (2000). For all problem classes they found that the (average) minimum number of setups generated by the ILSA was significantly smaller than the number of setups provided by KOMBI234, while the (average) amount of input was significantly larger. The same was true in comparison to SHP, which turned out to be dominated by KOMBI234, the latter providing solutions with fewer setups and smaller amounts of input (averages per problem class) than SHP. The average computing time per problem instance ranged between 0.05 and 70.25 seconds on a Pentium III/1GHz PC with 1 GB core memory.

In a subsequent paper (Umetani et al., 2006), the authors present an improved ILSA. As an additional feature, they introduce a second neighborhood structure (called shift neighborhood) which is applied alternately in the local search phase. It is defined by exchanging one small item in a cutting pattern with one or several other items from another pattern. After a local optimum has been found, the local search phase is started again from a randomly selected solution of the shift neighborhood. Results from numerical experiments demonstrate that this improved ILSA generates solutions with smaller amounts of trim loss for the same number of patterns.

Different to the model presented above, Umetani et al. (2003b) consider a variant of the SC_TMM_SU, in which they allow both surplus and shortage with respect to the demands, and they search to minimize the quadratic deviation of the provided small items from their respective demands for a given number $\max (s)$ of cutting patterns. It is interesting to note that their problem formulation does not explicitly consider the trim loss. For the stated problem, they present an algorithm (ILS-APG) which is based on Iterated Local Search. A neighborhood solution is obtained by replacing one cutting pattern in the current solution by another one, which is generated by an adaptive pattern generation technique. In order to evaluate the new solution, the application frequencies of the cutting patterns are determined by a heuristic based on the nonlinear Gauss-Seidel method.

The proposed method has been tested on the randomly generated instances of Foerster \& Wäscher (2000) and a set of instances taken from a practical application at a chemical fiber company in Japan and run against SHP and KOMBI234. The authors claim that their method provides comparable solutions, even though it must be remarked that the results are not completely comparable due to the fact that ILS-APG - unlike SHP and KOMBI234 - permits surplus and shortages.

\section{SETUP MINIMIZATION SUBJECT TO INPUT OR TRIM LOSS LIMITATIONS}

In contrast to the models and solution approaches presented in the previous section, one may minimize the number of setups subject to an acceptable level of input or trim loss.

\subsection{Input- or Trim Loss-Constrained Setup Minimization Models}

Let the following notation be introduced: 
$\max (o)$ : maximal number of large objects permitted in a solution;

$\max (t)$ : maximal (total) amount of trim loss permitted in a solution.

Then, on the basis of the IB_TCMM_SU, an Input-Constrained Setup Minimization Model (IC_SMM) can be obtained if we replace objective function (4.1) in model M 2.1 by

$$
\sum_{j \in J} \delta_{j} \rightarrow \operatorname{Min!}
$$

and add the following constraint:

$$
\sum_{j \in J} x_{j} \leq \max (o)
$$

Likewise, on the basis of the TB_TCMM_SU we receive a Trim Loss-Constrained Setup Minimization Model (TC_SMM) if we replace objective function (4.1) in model M 2.2 by (6.1) and add the constraint

$$
\sum_{j \in J} t_{j} \cdot x_{j} \leq \max (t)
$$

The problem of minimizing the number of cutting patterns (setups) subject to a given number of large objects or a given amount of trim loss is also called Pattern Minimization Problem (Vanderbeck, 2000). We point out that some authors particularly assume that the respective number of large objects or the respective amount of trim loss is minimal ( $c f$. McDiarmid, 1999, Alves $\&$ de Carvalho, 2008). This problem is strongly NP-hard, even for the special case that any two demanded items fit into the large object, but three do not (cf. McDiarmid, 1999).

\subsection{Solution Approaches}

Due to its NP-hardness, initially the Pattern Minimization Problem has usually been solved heuristically. We present these approaches first before we turn to more recent exact approaches.

\subsubsection{Heuristic Approaches}

Traditional methods of this kind start from of an (integer) input-minimal/trim loss-minimal solution and try to reduce the number of cutting patterns by recombining them in a series of steps without allowing for an increase in the input quantity/trim loss quantity. In this sense, these methods can be looked upon as lexicographic solution approaches. In each step the set of patterns is transformed into a set of smaller cardinality, and the corresponding application frequencies are adjusted such that all demands are still satisfied. The procedure, also called Pattern Reduction Technique ( $c f$. Foerster \& Wäscher, 2000) is terminated when no further combination is possible.

For the one-dimensional case, approaches of this kind have been suggested by Johnston (1986), Allwood \& Goulimis (1988), Goulimis (1990), and Diegel et al. (1996a). They have in common that they systematically try to replace pairs of patterns by a single pattern (2to1-combination) and triples of patterns by pairs (3to2-combination). The methods differ with respect to how the patterns to be combined are selected, and how the new (combined) patterns and their application frequencies are actually determined. KOMBI ( $c f$. Foerster, 1998; Foerster \& Wäscher, 2000) can 
be seen as a generalization of the method by Diegel et al. (1996a). It permits the combination of $p$ patterns into $q$ ( $p>q ; p, q$ integer; $q>2$ ) and is based on the fact that the sum of the application frequencies of the resulting patterns has to be identical to the sum of the frequencies belonging to the original patterns in order to keep the input constant. In computational experiments, which included 18 problem classes with 100 instances each, Foerster \& Wäscher (2000) demonstrated that KOMBI is superior to the other methods of this approach. However, since only a limited number of all possible $p$ to $q$-combinations are checked, it cannot be expected that a solution with a minimum number of patterns is found in the end, i.e. the final solution does not necessarily represent an optimum in the lexicographic sense.

Burkard \& Zelle (2003) consider a (one-dimensional) reel cutting problem in the paper industry which is characterized by several specific pattern constraints (maximum number of short items/reels in the pattern, maximum number of long items/reels in the pattern, minimum width of the pattern). They introduce a randomized local search (RLS) heuristic which is applied in two phases: In the first phase a near-optimal solution to the equality-constrained IMM is determined by means of move, swap (swap, swap $2 \times 1$, swap $2 \times 2$, swap $2 \times 1 \times 1$ exchanges) and normalization operations and additional "external" neighborhood moves. Since all feasible cutting patterns are considered, the solution is also near-optimal with respect to the trim loss. Then, in a second phase, the RLS heuristic - limited to a subset of the neighborhood moves - is applied in order to reduce the number of cutting patterns.

The authors have carried out numerical experiments with 6 problem instances from the paper industry. They have run their method against a software package which was used at the paper mill where the instances were obtained and achieved a significant reduction in the number of different patterns. (The authors also carried out additional experiments with the bin-packing instances from Falkenauer (1996) and from Scholl et al. (1997). The results are not reported here because the authors do not give any information on the quality of the solutions with respect to the number of patterns.)

\subsubsection{Exact Approaches}

The first exact approach to the (one-dimensional) Pattern Minimization Problem (PMP), a branch-and-price-and-cut algorithm for the one-dimensional case, was proposed by Vanderbeck (2000). An initial, compact formulation of the PMP is reformulated by means of the DantzigWolfe decomposition principle, resulting in an optimization problem where each column represents a feasible cutting pattern associated with a possible application frequency. This problem is solved by a column-generation technique in which the pricing (sub-) problem is a non-linear program. The non-linearity is overcome by solving a series of bounded knapsack problems. Cutting planes based on applying a superadditive function to single rows of the restricted master problem are used to strengthen the problem formulation. A branch-and-bound scheme guides the search of the solution space.

The algorithm was tested on 16 real-life problem instances. Only 12 instances (of small and medium size) could be solved to an optimum within two hours of CPU time on a HP3000/717/80 workstation. Clautiaux et al. (2010) later demonstrated that the function used by Vanderbeck for 
the generation of the cutting planes is not maximal and, therefore, may be dominated by other superadditive functions.

Alves \& de Carvalho (2008) present an exact branch-and-price-and-cut algorithm for the onedimensional PMP, which is based on Vanderbeck's model. The authors introduce a new bound on the waste, which strengthens the LP bound. It also reduces the number of columns to be generated and the corresponding number of knapsack problems to be solved in the columngeneration process. Dual feasible functions described by Fekete \& Schepers (2001) are used to derive valid inequalities superior to the cuts proposed by Vanderbeck. Furthermore, the authors formulate an arc-flow model of the problem which is used to develop a branching scheme for the branch-and-bound algorithm. Branching is performed on the variables of the model. The scheme avoids symmetry of the branch-and-bound tree.

Numerical experiments were run on a $3.0 \mathrm{Ghz}$ Pentium IV computer, the time limit was set to two hours again. The proposed algorithm solved one additional instance of Vanderbeck's set of test problem instances to an optimum. However, in comparison to Vanderbeck's algorithm, the number of branching nodes needed for finding an optimal solution was reduced by 46 percent for these instances. The LP bound obtained by adding the new cuts was also improved significantly. At the root node, the improvement amounted to 21.5 percent on average. The algorithm was further evaluated on 3,600 randomly generated test problem instances, divided into 36 classes of 100 instances each. The experiments clearly demonstrated that the percentage of optimally solved instances drops sharply with an increase in the number of small item types. For $m=40$ only one third of the instances could be solved to an optimum within 10 minutes of CPU time. For the remaining instances, the average optimality gap was 12.1 percent. Also, an increase in the average demand aver $(d)=\frac{1}{|I|} \sum_{i \in I} d_{i}$ reduces the percentage of optimally solved instances. This can be explained by the fact that an increase in $\operatorname{aver}(d)$ will increase the application frequencies which, again, will increase the number of pricing problems to be solved.

Based on a different model, Alves et al. (2009) develop new lower bounds for the one-dimensional PMP. The model itself is an integer programming model and can be solved by column generation; constrained programming and a family of valid inequalities are used to strengthen it. In order to verify the quality of the new bounds, numerical experiments were conducted on the benchmark instances of Vanderbeck (2000) and a subset of the (randomly generated) instances by Foerster \& Wäscher (2000). The results reveal clear improvements over the continuous bounds from Vanderbeck's model.

\section{MULTI-OBJECTIVE APPROACHES}

With respect to the non-availability of the relevant cost data, one might not be so much interested in finding a solution, which independently minimizes the amount of input, the amount of trim loss, or the number of setups, but which is "good" with respect to the amount of input or trim loss on one hand, and with respect to the number of setups on the other. This means that one will have to sacrifice some additional input or trim loss for a smaller number of cutting patterns. Corresponding approaches will be discussed in this section. 


\subsection{Bi-Objective Optimization Models}

Formally, in order to overcome the problem of the non-availability of the relevant cost data, each of the above-introduced total cost minimization models can be replaced by a corresponding biobjective optimization model. This gives rise to an Input-based Vector Optimization Model of the SSSCSP with Setups (IB_VOM_SU) which is obtained by substituting the objective function (4.1) in model M 2.1 by

$$
\left(\begin{array}{l}
\sum_{j \in J} x_{j} \\
\sum_{j \in J} \delta_{j}
\end{array}\right) \rightarrow \text { "Min!" }
$$

(cf. Jardim Campos \& Maculan, 1995, p. 45; Yanasse \& Limeira, 2006, p. 2745). For the respective Trim Loss-based Vector Optimization Model of the SSSCSP with Setups (TB_VOM _SU) we replace the objective function (4.6) of model 2.2 by

$$
\left(\begin{array}{c}
\sum_{j \in J} t_{j} \cdot x_{j} \\
\sum_{j \in J} \delta_{j}
\end{array}\right) \rightarrow \text { "Min!" }
$$

In (7.1) and (7.2), "Min!" refers to a minimization of the two-dimensional objective function in a vector-optimal sense and allows for several interpretations. Here, we first concentrate on the aspect that one wants to identify a single (compromise) solution. Afterwards, we deal with approaches which explore the entire set or a subset of all efficient solutions, from which the final cutting plan can be selected later.

\subsection{Approaches for the Determination of Compromise Solutions}

Approaches of this kind were the first ones described in the literature. They are based on RPE Techniques and differ with respect to what is considered to be an "acceptable" cutting pattern and how such patterns are generated.

Haessler (1975; also see Haessler, 1978, 1988) addresses the one-dimensional TB_VOM_SU and introduces the following aspiration levels that a cutting pattern $j$ must satisfy in order to be admitted into the cutting plan: maximum amount of trim loss per cutting pattern $\left(\max \left(t_{j}\right)\right)$, $\min$ imum and maximum number of small items per cutting pattern $\left(\min \left(\sum_{i \in I} a_{i j}\right), \max \left(\sum_{i \in I} a_{i j}\right)\right)$, and minimum number of times the pattern should be applied $\left(\min \left(x_{j}\right)\right) \cdot \min \left(\sum_{i \in I} a_{i j}\right)$, $\max \left(\sum_{i \in I} a_{i j}\right)$ and $\min \left(x_{j}\right)$ are calculated dynamically, based on the remaining demands. Small values of $\max \left(t_{j}\right)$ tend to provide cutting plans with a small amount of (total) trim loss while larger values for $\max \left(t_{j}\right)$ give solutions with a smaller number of different cutting patterns at the expense of additional trim loss. Haessler suggests to fix $\max \left(t_{j}\right)$ to values between 0.6 and 3.0 percent (Haessler, 1975, p. 487) or rather 0.5 and 2.0 percent (Haessler, 1978, p. 72) of the size of the stock objects. The search for a cutting pattern that satisfies all aspiration levels is carried out in a lexicographic order. If the search is not successful for the current set of aspiration levels, the aspiration level for the pattern frequency $\min \left(x_{j}\right)$ is reduced by one. In the case of $\min \left(x_{j}\right)=1$ the pattern with minimum trim loss is chosen in order to guarantee the termination of the algorithm. 
Vahrenkamp (1996) also considers the one-dimensional TB_VOM_SU. In the first place, his method is guided by two descriptors which define whether a pattern is acceptable for the cutting plan, namely (i) the percentage of trim loss which is tolerated for the pattern, and (ii) the minimum percentage of the open orders which should be cut by one pattern. The search for a pattern which satisfies the current aspiration levels for these descriptors is carried out by means of a randomized approach. Since it becomes more difficult to identify an acceptable pattern after each update of the demands, the aspiration levels will have to be relaxed in the search for a pattern. Relaxing the aspiration level for (ii) with priority will give cutting plans requiring a small number of stock objects, while giving priority to the relaxation of the aspiration level for (i) will result in cutting plans with a small number of different patterns. Interestingly, the decision on which aspiration level should be relaxed at a given point can be handed over to a random process, in which (i) is relaxed with probability prob and (ii) with probability 1-prob. This allows for a "mixing" (Vahrenkamp 1996, p. 195), i.e. a kind of weighting, of the two criteria of (6.2).

Yanasse \& Limeira (2006) examine the IB_VOM_SU. Their method is based on the idea that the demand of more than just one small item type should be satisfied when a pattern is cut according to its application frequency. Consequently, "good" patterns are those which complete the requirements of at least two small item types, and the algorithm tries to include such "good" patterns in the solution. The proposed method consists of three phases. In the first phase, cutting patterns are generated by means of the RPT. In particular, it is tried to compose patterns of small item types with similar demands or with demands which are multiples of each other. A pattern $j$ is only allowed to be included in the solution if it satisfies three aspiration levels, i.e. one with respect to the efficiency of the pattern $(\beta)$, one with respect to the application frequency of the pattern $\left(\operatorname{asp}\left(x_{j}\right)\right)$, and one with respect to whether the demand of more than one small item type will be satisfied if the pattern is cut $\operatorname{asp}\left(x_{j}\right)$ times. $\beta$ and $\operatorname{asp}\left(x_{j}\right)$ are computed dynamically from the data of the (initial/reduced) problem. In the second phase, when no more patterns are found which satisfy the aspiration levels, an input minimal cutting plan is determined for the residual problem. In the third phase, a pattern reduction technique is applied to reduce the number of patterns/setups. The authors remark that the final solution has proven to be sensitive to the choice of the cutoff value for $\beta$ and suggest rerunning their method with different values of this parameter.

The method can be applied to the IB_VOM_SU of any dimension. It has been evaluated on 18 classes of (one-dimensional) test problems, containing 100 instances each. The instances have been generated in a similar way as in Foerster \& Wäscher (2000). The results have been compared to the solutions provided by the method of Haessler (1975) and by KOMBI234 of Foerster \& Wäscher. With respect to the average objective function values per instance, the YanasseLimeira-Method (YLM) dominated Haessler's method on ten problem classes; for three problem classes the input was smaller while the number of setups was larger, and for the remaining five problem classes the number of setups was smaller while the input was larger for YLM. In comparison to KOMBI234, YML gave solutions with fewer setups for all problem classes at the expense of additional input. The average computing times per instance varied between the different classes from 0.12 to 50.61 seconds (on a Sun Ultra 30 workstation, $296 \mathrm{MHz}$ and 384 MB RAM). 
In Cerqueira \& Yanasse (2009), YLM is slightly modified with respect to how the cutting patterns are generated. For three classes of problem instances this method outperforms the original one, both in terms of the average input and the average number of patterns. For another 12 classes the authors obtained solutions with fewer setups but with more input.

Diegel et al. (2006) examine the continuous version of the one-dimensional IB_VOM_SU with a special emphasize on a problem environment encountered in the paper industry. They remark that a decrease in the number of setups can be achieved by increasing the length of the runs (i.e. the application frequencies) for some cutting patterns. Consequently the authors demonstrate how minimum run lengths can be enforced in a linear programming approach in order to reduce the number of setups. Their study is fundamental in nature; the authors do not indicate what solution should be selected and they do not present any insights from numerical experiments.

Dikili et al. (2007) present an approach to the IB_VOM_SU which is based on an explicit generation of all complete cutting patterns. From this set a pattern is selected by applying (in a lexicographic sense) the following selection criteria:

(i) minimum waste,

(ii) maximum frequency of application,

(iii) minimum total number of small items to be cut,

(iv) maximum number of the largest small item type.

The pattern is applied as often as possible without producing any oversupply. Afterwards, all patterns are eliminated from the pattern set which are no longer needed (because they include an item of which the demand is already satisfied) or which are infeasible (because the number of times a particular item type appears in a pattern exceeds its demand). The procedure stops when all demands are satisfied. The authors do not present any results from numerical experiments but express their ideas on two problem instances. The respective solutions include fewer setups than solutions obtained by linear programming. Due to the fact that all complete cutting patterns have to be generated initially, the presented heuristic will only be applicable to a limited range of problem instances.

SHPC, a sequential heuristic which has been proposed by Cui et al. (2008) for the one-dimensional TB_VOM_SU, determines the next pattern to be admitted into the cutting plan by means of a one-dimensional (bounded) knapsack problem in which only a subset (candidate set) of the remaining demands is considered. The authors argue that an increase in the number of item types and in the total length of the items in the candidate set will result in a reduction of the trim loss in the pattern to be generated. As a consequence, they control the composition of the candidate set by two parameters, namely (i) the minimum number $\min (m)$ of item types allowed in the pattern, and (ii) a multiple mult $(L)$ of the length $L$ of the large object which gives the minimum total length $\operatorname{mult}(L) \cdot L$ of the items in the candidate set.

Two additional parameters are introduced which are used for the definition of the item type values in the knapsack problem, i.e. (iii) $\alpha, 0 \leq \alpha \leq 1$, and (iv) $\beta, \beta \geq 1$. The item type values $v_{i}$ are then set as follows: $v_{i}=l_{i}$ if $l_{i} \leq \alpha \cdot L$, and $v_{i}=\beta \cdot L$ otherwise. This is motivated by the idea 
that longer items should be assigned to patterns at an early stage of the pattern generation process because assigning them at a late stage might result in poor material utilization. $\alpha$ defines what is taken as a long item, and $\beta$ determines the probability for a long item to be included in the pattern. A large value of $\alpha$ gives preference to the "really" long items, and a large value of $\beta$ increases the probability that they are included in the pattern. By means of numerical experiments the authors have identified the following appropriate values for $\alpha$ and $\beta: \alpha \in[0.4,0.5]$ and $\beta \geq 1.15$.

The generation of cutting plans is repeated for different values of $\max (m)$ and $r \cdot \max (m)$ is increased by one from a minimum to a maximum level, while for each $\max (m)$ value the $r$ value is increased at a constant rate from a minimum to a maximum level. The authors only record a single "best" cutting plan. The one which is currently stored as the best one is overridden if the new plan results in a smaller amount of trim loss or, otherwise, if it contains a smaller number of cutting patterns for the same amount of trim loss.

The authors have carried out numerical experiments in which they considered the 18 classes of randomly generated test problem instances of Foerster \& Wäscher (2000). The results have been compared to the solutions provided by KOMBI234 and YLM. As for the average trim loss and the average number of patterns, SHPC dominated KOMBI234 on 10 problem classes. For four classes it provided a smaller (average) amount of trim loss (at the expense of a larger average number of patterns) and for another four classes the average number of patterns was smaller (at the expense of a larger average amount of trim loss). KOMBI234 outperformed SHPC on one problem class. SHPC outperformed YLM with respect to both the average trim loss and the average number of patterns on five problem classes and provided solutions with a smaller average trim loss but with a larger average number of patterns for 12 classes. YLM outperformed SHPC on one class of problems. Average computing times per problem instance for SHPC were short; for the different classes they ranged between 0.16 and 1.42 seconds on a $2.80 \mathrm{GHz}$ PC with 512 MB core memory.

\subsection{Approaches for the Generation of Trade-Off Curves}

In order to explore the trade-off between input or trim loss on one hand, and the number of setups on the other, the above-described models SC_IMM_SU, SC_TMM_SU, IC_SMM and TC_SMM can be solved iteratively for different values of the right-hand sides of constraints (5.2), (6.2) and (6.3), respectively, which are systematically varied from respective upper bounds down to values for which no longer feasible solutions can be identified. By means of additional optimization steps it could even be ensured that this procedure will provide efficient (i.e. Pareto-optimal) solutions. However, long computing times may prohibit applying such approaches in practice.

Vasko et al. (2000; also see Vasko et al., 1992) describe MINSET ${ }^{\complement}$ - a software package for a one-dimensional TB_VOM_SU with demand tolerances in which the next pattern to be included in the cutting plan is determined by solving a (one-dimensional) knapsack problem. The small item types which may be included in the pattern/knapsack problem are limited to a certain subset of the (remaining) item types. In order to identify these item types, a candidate list is set up in which the item types are sorted in descending order based on the (remaining) demands. Then, in the given order, the small item types $i$ to be included in the knapsack problem are selected 
from the list until $\sum_{i=1}^{k} l_{i} \geq b . L$ holds for the first time, where $l_{i}$ represents the size (length) of the small item type $i$ and $L$ the size (length) of the large object. $k$ is the last item type which is admitted into the knapsack problem. The cutting pattern obtained from the knapsack problem is admitted into the cutting plan with a frequency of one if it is a new pattern. If the pattern already exists in the cutting plan then its frequency is increased by one. Afterwards, the demands are updated correspondingly. Furthermore, two descriptors are computed, namely (i) the sum of the current surplus and shortage in relation the total demand and (ii) the size of the unsatisfied demands in relation to the large object.

These steps are repeated until at least one of the descriptors satisfies a certain, pre-specified aspiration level, i.e. the descriptor has reached a level smaller than the corresponding aspiration level. The generation of cutting patterns is stopped at this stage, and one tries to identify cutting patterns which would reduce the sum of surplus and shortage if their frequency was increased. If such a pattern exists, the frequency of the one which would result in the largest reduction of surplus and shortage is increased by one. Otherwise, the algorithm terminates. $b$ is a parameter to be specified by the user of the program. It represents the (desired) degree of utilization of the large objects. Small values of $b$ tend to give solutions with few setups at the expense of larger trim loss, while large values of $b$ give solutions with a small amount of trim loss but more patterns/setups. Based on numerical experiments, the authors suggest that $b$ is varied in constant intervals of 0.1 from 0.2 to 1.3 . By doing so, a set of twelve solutions/cutting patterns is determined from which the non-dominated ones are presented to the planner as decision proposals. It cannot be guaranteed that these solutions are efficient with respect to the underlying TB_VOM_SU.

Vasko et al. (2000, p. 9) claim that solutions can be generated in 2 seconds or less on a $150 \mathrm{MHz}$ Pentium PC. They do not report any systematic numerical experiments, but - in order to demonstrate the trade-off between input and trim loss, and to exemplify their approach - they give the objective function values of the solutions they have obtained for four problem instances.

Umetani et al. (2003a, 2003b) also mention that their algorithms, ILS and ILS-APG, may be applied iteratively for different values of $\max (s)$, and they claim (Umetani et al., 2003a, p. 1094) that their numerical experiments have generated reasonable trade-off curves. The solutions provided are non-dominated ones. However, due to the heuristic nature of their algorithms, the efficiency of the solutions cannot be guaranteed.

Lee (2007) considers the one-dimensional TMM of the SSSCSP with demand tolerances. He formulates an integer bilinear programming model of the problem which holistically integrates the master problem (aiming at the fulfillment of the demands) and the subproblem for pattern generation. In each iteration, new patterns are generated "in situ", not "ex situ", like in the classic column-generation approaches to the SSSCSP and its variants. The author argues that a major drawback of classic column generation has to be seen in the fact that the prices used in the subproblem are based on a continuous solution of the master problem. They are not sufficient for this purpose since an integer solution of the underlying problem is required. Thus, particular attention is paid to the fact that the newly generated patterns give an improved integer solution rather than a continuous one. The proposed approach is imbedded in a heuristic framework which can be seen as a large neighborhood search. It allows for dealing effectively with constraints on 
the number of cutting patterns and can be used for the generation of a trade-off curve between the trim loss and the number of cutting patterns.

The heuristic, named CRAWLA, has been evaluated on 40 problem instances from chemical fiber production. The solution sets provided by CRAWLA were compared to those obtained by the ILS algorithm of Umetani et al. (2003a). It is interesting to note that, as for the limitations of the number of cutting patterns/setups, the two algorithms provide solution sets which cover different ranges. While CRAWLA provides a set of solutions for setups restricted to relatively small numbers, ILS instead gives solution sets in which the setups tend to be far less restricted but also miss out very small values for the constraints on the number of setups. In fact there is very little overlapping between the two ranges, but where they do overlap it becomes apparent that CRAWLA often dominates the solutions of ILS. According to the author, it took between one and twenty minutes to provide a set of solutions, but no information has been given on the utilized computer.

Golfeto et al. (2009b) have also modified their symbiotic genetic algorithm in order to provide a set of non-dominated solutions for the one-dimensional TB_VOM_SU. Their method, SYMBIO, has been applied to Umetani's 40 chemical fiber instances and compared to the results from CRAWLA and ILS. It can be seen that SYMBIO provides sets of solutions which cover a similar range of setups. Solution sets from SYMBIO are superior to those of CRAWLA for 17 instances, i.e. for each solution provided by CRAWLA another one provided by SYMBIO exists which is at least as good with respect to both the number of setups and the amount of trim loss, and there is at list one solution provided by CRAWLA which is dominated by a solution of SYMBIO. On the other hand, CRAWLA is superior to SYMBIO for 16 instances. For the remaining seven instances neither is SYMBIO superior (in the given sense) to CRAWLA, nor is CRAWLA superior to SYMBIO. The computing times seem to be a major drawback of SYMBIO. The authors reported between 40 and 60 minutes per instance on an AMD SEMPRON 2300+ PC (1.5 MHz, 640 MB RAM).

In Cui (2012), a decision support system is described into which a slightly modified version of SHPC is integrated. It offers an optional dialog in which the user is offered several nondominated cutting plans which have been generated when solving the respective cutting problem. The user can evaluate the cutting plans and select one of them for execution. It is worth noting that the system can also handle Multiple Stock Size Cutting Stock Problems (MSSCSP), i.e. problems with multiple types of large objects.

Kolen \& Spieksma (2000) consider an integrated cutting and setup problem for a slightly different one-dimensional problem setting. On the input side, the availability of the (identical) large objects is limited. On the output side, two types of demands can be distinguished, namely (i) demands which have to be satisfied exactly (exact orders), and (ii) demands, for which at least a given number of items must be provided (open orders). According to this setting, their problem can be categorized as a Multiple Identical Large Object Placement Problem (MILOPP). Furthermore, cutting-knife limitations have to be taken into account, i.e. the number of small items is limited which can be cut from a large object. The authors present a branch-and-bound algorithm that provides the entire set of Pareto-optimal solutions. 


\section{DISCUSSION}

In general, all models presented in this paper refer to a situation in which the cutting equipment represents a production stage which can be planned independently from other production stages. As for the total cost optimization (M 2.1, M 2.2) and the corresponding auxiliary models it is further assumed that the capacity of this stage is not fully utilized. In this case, the presented models can be considered as a fair representation of the practical situation. Nevertheless, according to our experience from practice, we believe that the actual situation is often misrepresented. In particular, the (relative) cost per setup ( $\mathrm{c}^{\text {SETUP }}$ ) appears to be frequently overestimated. What has to be noted is that this coefficient must only include the decision relevant costs, i.e. those costs which actually change when the number of setups varies. Imagine a cutting process in the steel industry, where the steel plates are passed beneath a scaffold on which a rim with a set of rotating knives is fixed. In order to change over from one pattern to another, the feeding of steel plates into the machine has to be stopped and the current rim has to be lifted from the scaffold (e.g. by a crane). The rim is then replaced by another one with a new setting of knives. Typically, this new rim has already been prepared in an outside workshop, where from it has to be moved to the cutting stage, e.g. by a forklift. Even though the entire change-over process appears to be rather elaborate and time-consuming, the decision-relevant costs are small, since the costs of the machinery (depreciation of the crane, forklift etc.) and the costs of labor (for the metal workers in the workshop, the operator of the crane, the driver of the forklift) must be considered fixed in the short planning period. Of course, some costs (e.g. energy costs of the forklift) may be identified as variable and, consequently, as decision relevant, but they are usually small in relation to the cost of the input ( $\mathrm{c}^{\mathrm{INPUT}}$ ), e.g. the material cost, or the cost of trim loss (cTRIM). From this we conclude that approaches which minimize the number of cutting patterns subject to minimal input (models of the IC_SMM type) or minimal trim loss (TC_SMM) are - to a certain extent actually justified. Consequently, we suggest that in numerical experiment also sets of problem instances are considered which contain instances where the cost of the input or the cost of the trim loss is sufficiently larger than the setup cost.

The fact that the (relative) setup costs are frequently overestimated may be related to situations in which the capacity of the cutting stage is fully utilized. In this case, practitioners tend to introduce opportunity costs as setup costs in order to value the loss of productive time caused by the setup process. From a theoretical point of view, this is a rather unsatisfying approach, since opportunity costs should be part of the model output, not of the input. More convincing would be a modeling approach that explicitly takes into account for limitations of the capacity of the cutting stage and its utilization by cutting and setup processes. However, no models (and also methods) for cutting problems with setups exist so far which consider such capacity constraints.

\section{OUTLOOK: RESEARCH OPPORTUNITIES}

In this section we would like to point out a few opportunities for future research. According to what has been said in the previous section, we acknowledge that there is still room for further research. 
We have already noticed the limited range of problem types which have been treated in the literature so far. Of the problem types related to input minimization, usually only problems with a single stock size are considered, in contrast to problems with multiple stock sizes which only have been dealt with occasionally (Cui, 2012). Literature related to problems of the outputmaximization type is - with the exception of Kolen \& Spieksma (2000) - practically nonexisting. As for the dimension of the problem types, research has concentrated on one-dimensional problems. Apart from Farley \& Richardson (1984), two-dimensional cutting problems have almost been ignored completely. Thus we conclude that in future research could concentrate on those problem types which have been neglected so far.

Industrial cutting problems are often imbedded in a production environment which is significantly different to the basic one assumed in the models introduced above. Several (parallel) cutting machines may be available (Gilmore \& Gomory, 1963; Menon \& Schrage, 2002), not only different with respect to capacity, speed and cost of cutting, but also with respect to setup times and cost. In this case, the determination of cutting patterns (and setups) and the assignment of patterns to machines will be strongly affiliated with each other. The cutting process may also be distributed over two or more stages (Haessler, 1979, Zak, 2002a, 2002b). Integrating such aspects into existing models and algorithms or developing new ones which are capable to deal with such aspects appears to be a worthwhile effort with respect to practical applications.

As mentioned before, model extension related to capacity constraints would be another obvious area into which research could evolve. Here the question will have to be addressed what assumptions have to be made with respect to those orders (small items) which cannot be accommodated in the current period. In other words, the inclusion of capacity constraints would immediately lead to multi-period models which comprise due-dates and out-of-stock costs (costs of delayed shipments and/or lost sales costs). Additional aspects to be included in such models could further be related to inventory and probably lot sizing.

A very straightforward modification of the presented models would consist of allowing for pattern-dependent or even pattern sequence-dependent setup costs. The latter aspect gives rise to the Pattern Sequencing Problem (Pierce, 1970). Belov \& Scheithauer (2007) present an approach in which the goal of the latter problem is represented by the minimization of the number of open stacks (Yuen, 1991, 1995; Yuen \& Richardson, 1995; Yanasse et al., 1999). So do Matsumoto et al. (2011) for the MSSCSP; the authors additionally constrain the number of setups for type of large object. Alternative, practically relevant goals of the Pattern Sequencing Problem (see Foerster, 1998), like the minimization of the number of cutting discontinuities (Dyson \& Gregory, 1974; Madsen, 1988; Haessler, 1992), the number of idle stacks (Nordström \& Tufekci, 1994) or the maximum order spread (Madsen, 1988), have not been considered in this respect.

Finally, an aspect widely ignored in the area of cutting and packing in general is uncertainty. The only study which addresses this aspect with respect to integrated cutting and setup problems so far (Beraldi et al., 2009) considers the case of demand uncertainty. The authors point out that solutions from deterministic models will typically result in overproduction and/or profit reduction. They develop a two-stage, non-linear, highly non-convex stochastic programming model 
which is based on a scenario approach. They also demonstrate that standard software is only able to solve problem instances of very limited size. It becomes obvious that there is a lack of approaches which can efficiently deal with demand uncertainty in real world integrated cutting and setup problems. Also approaches to other kind of uncertainties (e.g. yield uncertainty) still have to be developed.

\section{REFERENCES}

[1] Allwood JM \& Goulimis CN. 1988. Reducing the number of patterns in one-dimensional cutting stock problems. Control Section Report No. EE/CON/IC/88/10, Department of Electrical Engineering, Industrial Systems Group, Imperial College, London SW7 2BT (June).

[2] Alves C \& Valério de Carvalho JM. 2008. A branch-and-price-and cut algorithm for the pattern minimization problem. RAIRO - Operations Research, 42: 435-453.

[3] Alves C, Macedo R \& Valério de Carvalho JM. 2009. New lower bounds based on column generation and constraint programming for the pattern minimization problem. Computers and Operations Research, 36: 2944-2954.

[4] ATKIN T \& ÖZDEMIR RG. 2009. An integrated approach to the one-dimensional cutting stock problem in coronary stent manufacturing. European Journal of Operational Research, 196: 737-743.

[5] Belov G \& Scheithauer G. 2007. Setup and open-stacks minimization in one-dimensional stock cutting. INFORMS Journal on Computing, 19: 27-35.

[6] Beraldi P, BRUNi ME \& CONFORTI D. 2009. The stochastic trim-loss problem. European Journal of Operational Research, 197: 42-49.

[7] Burkard RE \& Zelle C. 2003. A local search heuristic for the reel cutting problem in paper production. Bericht Nr. 257, Spezialforschungsbereich F 003 - Optimierung und Kontrolle, Projektbereich Diskrete Optimierung, Karl-Franzens-Universität Graz und Technische Universität Graz (Februar).

[8] Cerqueira GRL \& Yanasse HH. 2009. A pattern reduction procedure in a one-dimensional cutting stock problem by grouping items according to their demands. Journal of Computational Interdisciplinary Sciences, 1(2): 159-164.

[9] Clautiaux F, Alves C \& Valério de Carvalho JM. 2010. A survey of dual-feasible and superadditive functions. Annals of Operations Research, 179: 317-342.

[10] CUI Y. 2012. A CAM system for one-dimensional stock cutting. Advances in Engineering Software, 47: 7-16.

[11] Cui Y, Zhao X, YANG Y \& YU P. 2008. A heuristic for the one-dimensional cutting stock problem with pattern reduction. Proceedings of the Institution of Mechanical Engineers, Part B: Journal of Engineering Manufacture, 222: 677-685.

[12] DEMIR MC. 2008. A pattern generation-integer programming based formulation for the carpet loading problem. Computers \& Industrial Engineering, 54: 110-117.

[13] Diegel A, Chetty M, van Schalkwyk S \& Naidoo S. 1996a. Setup combining in the trim los problem. Working paper, 7th draft, Business Administration, University of Natal, Durban. 
[14] Diegel A, Montocchio E, Walters E, van Schalkwyk S \& Naidoo S. 1996b. Setup minimising conditions in the trim loss problem. European Journal of Operational Research, 95: 631-640.

[15] Diegel A, Miller G, Montocchio E, van Schalkwyk S \& Diegel O. 2006. Enforcing minimum run length in the cutting stock problem. European Journal of Operational Research, 171: 708-721.

[16] Dikili AC, SariöZ E \& Pek NA. 2007. A successive elimination method for one-dimensional stock cutting problems in ship production. Ocean Engineering, 34: 1841-1849.

[17] DyckHOFF H. 1981. A new linear programming approach to the cutting stock problem. Operations Research, 29: 1092-1104.

[18] DyCKhOfF H. 1988. Production-theoretic foundation of cutting and related processes. Essays on Production Theory and Planning (FAndel G, DyCKhoff H \& REESE J. (Eds.)). Berlin et al:: Springer, $151-180$.

[19] Dyson RG \& GREgORY AS. 1974. The cutting stock problem in the flat glass industry. Operations Research Quarterly, 25: 41-54.

[20] FalKenauer E. 1996. A hybrid grouping genetic algorithm for bin packing. Journal of Heuristics, 2: 5-30.

[21] FARley AA \& Richardson KV. 1984. Fixed charge problems with identical fixed charges. European Journal of Operational Research, 18: 245-249.

[22] Fekete S \& SChepers J. 2001. New classes of fast lower bounds for bin packing problems. Mathematical Programming, 91: 11-31.

[23] Foerster H. 1998. Fixkosten- und Reihenfolgeprobleme in der Zuschnittplanung. Frankfurt am Main: Peter Lang.

[24] Foerster H \& Wëscher G. 1998. Simulated annealing for order spread minimization in sequencing cutting patterns. European Journal of Operational Research, 110: 272-281.

[25] Foerster H \& WÄscher G. 2000. Pattern reduction in one-dimensional cutting stock problems. International Journal of Production Research, 38: 1657-1676.

[26] Gilmore PC \& GOMORY RE. 1961. A linear programming approach to the cutting-stock problem. Operations Research, 9: 849-859.

[27] Gilmore PC \& GOMORY RE. 1963. A linear programming approach to the cutting-stock problem - Part II. Operations Research, 11: 864-888.

[28] Gilmore PC \& Gomory RE. 1965. Multi-stage cutting problems of two or more dimensions. Operations Research, 13: 94-120.

[29] Golfeto RR, Moretti AC \& Salles Neto LL. 2009a. A genetic symbiotic algorithm applied to the one-dimensional cutting stock problem. Pesquisa Operacional, 29: 365-382.

[30] Golfeto RR, Moretti AC \& Salles Neto LL. 2009b. A genetic symbiotic algorithm applied to the cutting stock problem with multiple objectives. Advanced Modelling and Optimization, 11: 473-501.

[31] Goulimis C. 1990. Optimal solutions for the cutting stock problem. European Journal of Operational Research, 44: 197-208. 
[32] HAESSLER RW. 1975. Controlling cutting pattern changes in one-dimensional trim problems. Operations Research, 23: 483-493.

[33] HAessler RW. 1978. A procedure for solving the 1.5-dimensional coil slitting problem. AIIE Transactions, 10: 70-75.

[34] Haessler RW. 1979. Solving the two-stage cutting stock problem. Omega, 7: 145-151.

[35] HAessler RW. 1988. Selection and design of heuristic procedures for solving roll trim problems. Management Science, 34: 1460-1471.

[36] HAessLeR RW. 1992. One-dimensional cutting stock problems and solution procedures. Mathematical and Computer Modelling, 16: 1-8.

[37] Haessler RW \& TALBOT B. 1983. A 0-1 model for solving the corrugator trim problem. Management Science, 29: 200-209.

[38] HajizAdeh I \& LeE C-G. 2007. Alternative configurations for cutting machines in a tube cutting mill. European Journal of Operational Research, 183: 1385-1396.

[39] Helmberg C. 1995. Cutting aluminium coils with high length variabilities. Annals of Operations Research, 57: 175-189.

[40] JaRdim CAMpos MH \& Maculan N. 1995. Optimization problems related to the cut of paper reels: A dual approach. Investigación Operativa, 5(1): (April), 45-53.

[41] Johnston RE. 1986. Rounding algorithms for cutting stock problems. Asia-Pacific Journal of Operational Research, 3: 166-171.

[42] Kolen AW J \& SPIEKSMA FCR. 2000. Solving a bi-criterion cutting stock problem with open-ended demand: a case study. Journal of the Operational Research Society, 51: 1238-1247.

[43] Koch S, KöNIG S \& WÄscher G. 2009. Integer Linear Programming for a Cutting Problem in the Wood-Processing Industry: A Case Study. In: International Transactions in Operational Research, 16: $715-726$.

[44] LEE J. 2007. In situ column generation for a cutting-stock problem. Computers \& Operations Research, 34: 2345-2358.

[45] Li S. 1996. Multi-job cutting stock problems with due dates and release dates. Journal of the Operational Research Society, 47: 490-510.

[46] Madsen OBG. 1988. An application of the travelling-salesman routines to solve pattern-allocation problems in the glass industry. Journal of the Operational Research Society, 39: 249-256.

[47] Matsumoto K, Umetani J \& Nagamochi H. 2011. On the one-dimensional cutting stock problem in the paper tube industry. Journal of Scheduling, 14: 281-290.

[48] MCDiarmid C. 1999. Pattern minimisation in cutting stock problems. Discrete Applied Mathematics, 98: $121-130$.

[49] Menon S \& Schrage L. 2002. Order allocation for stock cutting in the paper industry. Operations Research, 50: 324-332.

[50] Moretti AC \& Salles Neto LL. 2008. Nonlinear cutting stock problem model to minimize the number of different patterns and objects. Computational \& Applied Mathematics, 27(1): 61-78.

[51] Neumann K \& Morlock M. 1993. Operations Research. München, Wien: Hanser. 
[52] NONÅS SL \& THORSTENSON A. 2000. A combined cutting-stock and lot-sizing problem. European Journal of Operational Research, 120: 327-342.

[53] Nordström A-L \& TUfEKCI S. 1994. A genetic algorithm for the talent scheduling problem. Computers and Operations Research, 21: 927-940.

[54] Pegels CC. 1967. A comparison of scheduling models for corrugator production. The Journal of Industrial Engineering, 18: 466-471.

[55] PIERCE JF. 1964. Some large-scale production scheduling problems in the paper industry. Englewood Cliffs (N.J.): Prentice Hall.

[56] PierCe JF. 1970. Pattern sequencing and matching in stock cutting operations. Tappi, 53: 668-678.

[57] RAO MR. 1976. On the cutting stock problem. Journal of the Computer Society of India, 7: 35-39.

[58] Reinertsen H \& Vossen TWM. 2010. The one-dimensional cutting stock with due dates. European Journal of Operational Research, 201: 701-711.

[59] Rodriguez MA \& Vecchietti A. 2012. Integrated Planning and Scheduling with Due Dates in the Corrugated Board Boxes Industry. Industrial \& Engineering Chemistry Research, 52: 847-860.

[60] Schilling G \& Georgiadis MC. 2002. An algorithm for the determination of optimal cutting patterns. Computers \& Operations Research, 29: 1041-1058.

[61] Scholl A, Klein R \& JÜrgens C. 1997. A fast hybrid procedure for exactly solving the onedimensional bin packing problem. Computers \& Operations Research, 24: 627-645.

[62] TERLAKY T. 1985. A convergent criss-cross method. Mathematische Operationsforschung und Statistik-Series Optimization, 16: 683-690.

[63] Umetani S, Yagiura M \& Ibaraki T. 2003a. An LP-based local search to the one dimensional cutting stock problem using a given number of cutting patterns. IEICE Transactions on Fundamentals of Electronics, Communications and Computer Sciences, E86-A: 1093-1102.

[64] Umetani S, YagiURa M \& IBARaKi T. 2003b. One-dimensional cutting stock problem to minimize the number of different patterns. European Journal of Operational Research, 146: 388-402.

[65] Umetani S, Yagiura M \& Ibaraki T. 2006. One-dimensional cutting stock problem with a given number of setups: A hybrid approach of metaheuristics and linear programming. Journal of Mathematical Modelling and Algorithms, 5: 43-64.

[66] Vahrenkamp R. 1996. Random search in the one-dimensional cutting stock problem. European Journal of Operational Research, 95: 191-200.

[67] VAlÉRIO de CARVAlHo JM. 1998. Exact solutions of cutting stock problems using column generation and branch-and-bound. International Transactions in Operational Research, 5: 35-44.

[68] VANDERBECK F. 2000. Exact algorithm for minimizing the number og setups in the one-dimensional cuttingstock problem. Operations Research, 48: 915-926.

[69] Vasko F, Newhart D, Stott K \& Wolf F. 2000. Fiddler on the roof - Balancing trim loss and setups. OR Insight, 13(3): (July-September), 9-14.

[70] Vasko FJ, Wolf FE, Stott KL \& Ehrsam O. 1992. Bethlehem Steel combines cutting stock and set covering to enhance customer service. Mathematical and Computer Modelling, 16: 9-17. 
[71] VASKo FJ, Newhart DD \& STOTT KL. 1999. A hierarchical approach for one-dimensional cutting stock problems in the steel industry that maximizes yield and minimizes overgrading. European Journal of Operational Research, 114: 72-82.

[72] Wëscher G \& GAU T. 1996. Heuristics for the integer one-dimensional cutting stock problem: A computational study. OR Spectrum, 18: 131-144.

[73] Wäscher G, Haussner H \& Schumann H. 2007. An Improved Typology of Cutting and Packing Problems. European Journal of Operational Research, 183: 1109-1130.

[74] YANASSE HH. 1997. On a pattern sequencing problem to minimize the maximum number of open stacks. European Journal of Operational Research, 100: 454-463.

[75] Yanasse HH, BeCCeneri JC \& Soma NY. 1999. Bounds for a problem of sequencing patterns. Pesquisa Operacional, 19: 249-278.

[76] YANASSE HH \& LimEIRA MS. 2006. A hybrid heuristic to reduce the number of different patterns in cutting stock problems. Computers \& Operations Research, 33: 2744-2756.

[77] YUEN BJ. 1991. Heuristics for sequencing cutting patterns. European Journal of Operational Research, 55: 183-190.

[78] YUEN BJ. 1995. Improved heuristics for sequencing cutting patterns. European Journal of Operational Research, 87: 57-64.

[79] YUEN BJ \& RiCHARDSON KV. 1995. Establishing the optimality of sequencing heuristics for cutting stock problems. European Journal of Operational Research, 84: 590-598.

[80] ZAK EJ. 2002a. Modeling multistage cutting stock problems. European Journal of Operational Research, 141: 313-327.

[81] ZAK EJ. 2002b. Row and column generation technique for a multistage cutting stock problem. Computers \& Operations Research, 29: 1143-1156. 